\title{
Logarithm of the odds (LOD) score
}

National Cancer Institute ( $\mathrm{NCl})$

\section{Source}

National Cancer Institute (NCI). Logarithm of the odds (LOD) score.

A statistical estimate of whether two genetic loci are physically near enough to each other (or "linked") on a particular chromosome that they are likely to be inherited together. A log arithm of the odds score of 3 or higher is generally understood to mean that two genes are located close to each other on the chromosome. In terms of significance, a logarithm of the odds score of 3 means the odds are 1,000:1 that the two genes are linked and therefore inherited together. Also called LOD score. 